\title{
New BRC neutron evaluations of actinides with the TALYS code: Modelization and first validation tests
}

\author{
B. Morillon, H. Duarte, and P. Romain ${ }^{\mathrm{a}}$
}

CEA, DAM, DIF, 91297 Arpajon, France

Abstract. The reader may have a look on references [1-3,5] for more details.

Over the last five years, new evaluations of plutonium and uranium have been performed at Bruyères-le-Châtel (BRC) from the resolved resonance region up to $30 \mathrm{MeV}$. Only nuclear reactions models have been used to build these evaluations. Total, shape elastic and direct inelastic cross sections are obtained from a coupled channel model using a dispersive optical potential (BRC, [1-3]) devoted to actinides. All the other cross sections are calculated owing to the Hauser-Fesbach theory (TALYS code [4]). We take particular care over the fission channel. For uranium isotopes, a triple-humped barrier [3] is required in order to reproduce accurately the variations of the experimental fission cross sections. As not commonly expected, we show [5] that the effect of the class II or class III states located in the wells of the aforementioned fission barrier provide sometimes an anti-resonant transmission rather than a resonant. With increasing neutron incident energy, a lot of residual nuclei produced by nucleon emission lead to fission also. All available experimental data assigned to the various fission mechanisms of the same nucleus are used to define its fission barrier parameters. As a result of this approach, we are now able to provide consistent evaluations for a large series of isotopes. Of course, our new evaluations have been tested against integral data.

\section{References}

1. P. Romain and J.-P. Delaroche, "A dispersive coupled channels analysis of nucleon scattering from ${ }^{181} \mathrm{Ta}$ and ${ }^{182,184,186} \mathrm{~W}$ up to $200 \mathrm{MeV}$ " Proceedings of the NEA Specialists' Meeting on the Nucleon-Nucleus Optical Model up to 200 MeV, Bruyères-le-Châtel (1996) (OECD, Paris, 1997), (http//db. nea.fr/html/science/om200/), p. 167.

2. B. Morillon and P. Romain, Phys. Rev. C 70, 014601 (2004).

3. M.J. López Jiménez, B. Morillon and P. Romain, Ann. Nucl. Energy 32, 195 (2005).

4. A.J. Koning, S. Hilaire and M.C. Duijvestijn, "TALYS-1.0", Proceedings of the International Conference on Nuclear Data for Science and Technology - ND2007, (EDP Sciences Volume 1, eds. O. Bersillon, F. Gunsing, E. Bauge, R. Jacqmin and S. Leray, Apr. 22-27, 2007, Nice, France, 2008), p. 211.

5. B. Morillon, H. Duarte and P. Romain, "New BRC neutron evaluations of actinides with the TALYS code: modelization and first validation tests", Proceedings of the International Conference on Nuclear Data for Science and Technology - ND2007 (AIP Conference Proceedings 1175, eds. A. Chatillon, H. Faust, G. Fioni, D. Goutte and H. Goutte, May 13-16, 2009, Cadarache, France, 2009), p. 79.

\footnotetext{
a e-mail: pascal.romain@cea.fr
}

This is an Open Access article distributed under the terms of the Creative Commons Attribution-Noncommercial License 3.0, which permits unrestricted use, distribution, and reproduction in any noncommercial medium, provided the original work is properly cited. 